\title{
Zero TE MRI for Craniofacial Bone Imaging
}

\author{
(1) A. Lu, ${ }^{-}$K.R. Gorny, and $\mathbb{P}_{\text {M. }}$-L. Ho
}

\section{ABSTRACT}

SUMMARY: Zero TE MR imaging is a novel technique that achieves a near-zero time interval between radiofrequency excitation and data acquisition, enabling visualization of short-T2 materials such as cortical bone. Zero TE offers a promising radiation-free alternative to CT with rapid, high-resolution, silent, and artifact-resistant imaging, as well as the potential for "pseudoCT" reconstructions. In this report, we will discuss our preliminary experience with zero TE, including technical principles and a clinical case series demonstrating emerging applications in neuroradiology.

ABBREVIATIONS: $\mathrm{RF}=$ radiofrequency; $\mathrm{SAR}=$ specific absorption rate; $\mathrm{ZTE}=$ zero $\mathrm{TE}$

C hildren in the United States undergo $>5$ million diagnostic CT examinations per year. For certain indications (eg, acute screening and cortical bone assessment), CT remains a first-line technique. However, adverse effects from radiation are of great concern and include both deterministic (developmental disruption, skin damage, cataracts, sterility, radiation sickness) and stochastic effects (cancer, hereditary defects, growth impairment). These effects are magnified in children due to the increased sensitivity of developing tissues/organs, predisposing conditions that increase susceptibility, and greater cumulative lifetime risk of radiation exposure. ${ }^{1}$ Concerns regarding the adverse effects of radiation in the pediatric population have prompted the "Image Gently" campaign. ${ }^{2}$

Received March 6, 2019; accepted after revision July 8.

From the Department of Radiology, Nationwide Children's Hospital (M.-L.H.), The Ohio State University College of Medicine, Columbus, Ohio; and Department of Medical Physics (A.L., K.R.G.), Mayo Clinic, Rochester, Minnesota.

This work was supported by a Siemens/Radiological Society of North America Scholar Grant, a Society for Pediatric Radiology Pilot Award, and an American Society of Head and Neck Radiology William N. Hanafee Research Grant. Poster previously presented at: Annual Meeting of the International Society for Magnetic Resonance in Medicine, April 22-27, 2017; Honolulu, Hawaii. Work was pertinent to the article, but not representing the majority of content. Improved Delineation of Air-Bone Interface in In-Vivo High-Resolution Bright Bone ZTE MRI at 3T. Electronic exhibit previously presented at: Annual Meeting of the Radiological Society of North America, November 25-30, 2018; Chicago, Illinois. Improved Imaging of Craniosynostosis with Cinematic Rendering and ZTE MRI.

Please address correspondence to Mai-Lan Ho, MD, Department of Radiology, Nationwide Children's Hospital, The Ohio State University College of Medicine, Columbus, OH, 700 Children's Dr, ED4, Columbus, OH 43205; e-mail: mailanho@gmail.com

Indicates article with supplemental on-line photos.

http://dx.doi.org/10.3174/ajnr.A6175
Zero TE (ZTE) MR imaging has recently emerged as a promising alternative technique that achieves a near-zero time interval between the end of the radiofrequency (RF) excitation and the start of data acquisition. Unlike the conventional RF pulse sequences, the ZTE sequence begins a readout period immediately after the RF pulse, enabling visualization of short-T2 materials (ie, on the order of $\leq 1 \mathrm{~ms}$ ) such as cortical bone (On-line Fig 1). ${ }^{3-6}$ The ZTE sequence is rapid, high-resolution, silent, and resilient to artifacts caused by motion and $\mathrm{B}_{0}$ inhomogeneitycharacteristics that are well-suited to pediatric use..$^{7-9}$ Raw images demonstrate relative signal void compared with surrounding soft tissues, colloquially known as a "black-bone" display. There is computational potential for "bright-bone" reconstructions that are visually analogous to CT (Figure). ${ }^{10,11}$ Prior work has demonstrated the promise of ZTE and its related techniques, ultrashort$\mathrm{TE}$ and gradient-echo MR imaging, in bone imaging applications, including craniofacial, temporal bone, jaw, and spine. ${ }^{12-17}$ Small clinical case series and ex vivo studies have partially validated ZTE for cranial vault, trauma, and bone microstructural imaging. ${ }^{18,19}$ With ongoing MR imaging technical advances enabling ever-faster scan times and higher contrast/spatial resolution, there is a potential for this single technique to provide comprehensive imaging assessment for patients, a "one-stop shop." In this report, we discuss our initial experience with ZTE and provide multiple examples demonstrating the diagnostic utility of the sequence for craniofacial bone imaging.

\section{MATERIALS AND METHODS Technical Considerations}

In ZTE, readout gradients are turned on before the RF pulse so that data acquisition can begin immediately after excitation. This 

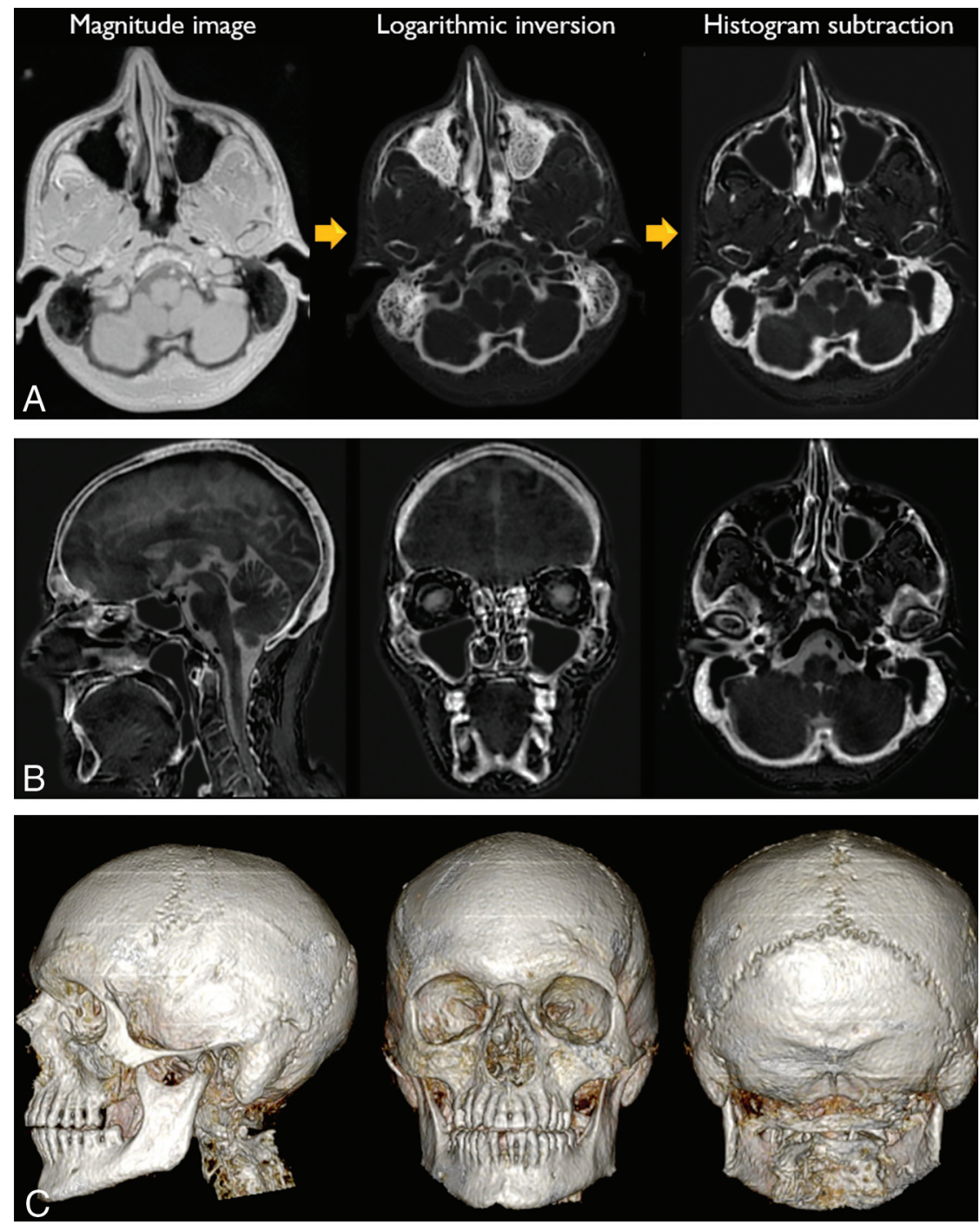

FIGURE. ZTE image display. A, Raw magnitude black-bone images underwent signal intensity correction on a section-by-section basis with normalization to mean soft-tissue signal. Multi-resolution ROI-based correction was then applied to account for residual signal intensity variations. Logarithmic inversion of the intensity-corrected data yielded bright-bone images. Histogram subtraction was used to identify and mask the peak signal of air, thus generating the final pseudoCT image. B, Sample sagittal, coronal, and axial reformats of pseudoCT reconstruction. $C, 3 D$ volume-renderings were generated at a separate workstation.

acquisition. Reduction of total scan time is achieved with the use of short TRs and small flip angles for quasisteady-state magnetization. Most scan time is targeted toward data acquisition, with minimal dead time occurring during the RF pulse and a single gradient step (On-line Fig 1). ${ }^{3-6}$ This feature permits rapid isotropic scanning with high resistance to artifacts, including susceptibility and motion. The technique uses lower changes in amplitude over time $(\mathrm{dB} / \mathrm{dt})$ than conventional sequences; low flip angles and built-in scanner safety measures counter the short RF pulse duration that would otherwise raise concern for a high specific absorption rate (SAR). ${ }^{9}$

\section{Patient Indications}

Imaging examinations (CT and MR imaging) were ordered by referring physicians for various diagnostic indications, including craniosynostosis, trauma, head and neck cancer, radiation therapy, and surgical planning and follow-up. Sedation or anesthesia or both were used for imaging only when deemed medically necessary by the referring clinician. In patients undergoing $\mathrm{MR}$ imaging, ZTE images of the whole head were acquired using FDAapproved product hardware (8-channel brain coil array; GE Healthcare, Milwaukee, Wisconsin) and software (Silenz; GE Healthcare). ${ }^{7,8}$ Imaging was preferentially performed at $3 \mathrm{~T}$ (Discovery MR750w, DV 26.0 software; GE Healthcare), except for patients with MR imaging-conditional devices necessitating imaging at $1.5 \mathrm{~T}$ (Optima MR450w, DV 26.0 software; GE Healthcare). The ZTE sequence had the

requires high-performance coils with rapid transmit/receive switching capabilities and uses center-out $k$-space encoding. With ZTE, the readout gradient is already on during the RF pulse, precluding conventional section/slab selection. This necessitates a high-bandwidth excitation with a short-duration hard pulse to ensure that the excitation is as homogeneous and as consistent as possible across the entire FOV from repetition to repetition. The RF excitation is immediately followed by a $3 \mathrm{D}$ data acquisition. Gradients are used continuously and modulated between repetitions to sample data along radial trajectories in $k$-space, rather than being rapidly turned on and off. This process minimizes mechanical vibration and enables a virtually silent technique. Fully sampling the $k$-space with radial trajectories requires significantly more repetitions (TRs) than an equivalent 3D Cartesian following parameters: 3D imaging mode with sagittal acquisition; $\mathrm{TE}=0.016 \mathrm{~ms}, \mathrm{FOV}=18 \times 18 \mathrm{~cm}$, readout matrix $=180 \times 180 \times$ 180 , section thickness $=1 \mathrm{~mm}, \mathrm{NEX}=1$, receiver bandwidth $=$ $31.25 \mathrm{kHz}$, flip angle $=2^{\circ}$. These yielded whole-head coverage at a $1-\mathrm{mm}$ isotropic voxel size. Sequence scan time was 2 minutes at $3 \mathrm{~T}$ and 2 minutes 30 seconds at $1.5 \mathrm{~T}$. Two separate data acquisitions were obtained in each patient, for a total imaging time of 4-5 minutes. We used 2 relatively short acquisitions rather than a single acquisition with a higher number of averages to reduce the overall impact of patient motion. All SAR measurements were confirmed to be within FDA safety limits based on predetermined scanner settings. The institutional review board at Mayo Clinic approved retrospective review and analysis of CT and MR images. 


\section{Image Postprocessing}

By means of a novel technique developed at our institution, source images underwent postprocessing using an off-line Matlab code (MathWorks, Natick, Massachusetts) to improve bone-air differentiation and generate "bright-bone" reconstructions visually analogous to CT. ${ }^{20}$ For most patients, both ZTE acquisitions were averaged to achieve better SNR after rigid body registration. In cases where $1 \mathrm{ZTE}$ acquisition was visibly distorted by motion and the other was not, only the acquisition with less motion was used for postprocessing, albeit with a lower total SNR. Raw magnitude images underwent signal intensity correction on a section-by-section basis, with normalization to mean soft-tissue signal within each section. Multiresolution ROI-based intensity correction was then applied to account for residual signal intensity variations and thus reduce overall image noise. ${ }^{21}$ Logarithmic inversion of the intensity-corrected data yielded preliminary bright-bone images, but without optimal bone-air delineation. An intensity histogram was generated and an intensity mask was selected to remove the peak signal of air, generating the final pseudoCT image and enabling generation of $3 \mathrm{D}$ volume-renderings at a separate workstation (Fig 1).

\section{Case Series}

Fourteen patients ( 2 months to 17 years of age) were successfully imaged. The following examples demonstrate that ZTE was qualitatively comparable with CT for pre- and postoperative assessment of bony abnormalities of the skull, face, and craniocervical junction. The additional value of MR imaging was provided for brain parenchymal and soft-tissue characterization in multiple instances.

Patient 1 was a 3-month-old boy with left anterior plagiocephaly. CT and ZTE comparably demonstrated premature fusion of the left coronal suture with associated calvarial flattening and harlequin eye deformity. The remaining cranial sutures were confirmed to be patent (On-line Fig 2).

Patient 2 was a 7-month-old girl with Apert syndrome. CT and ZTE both demonstrated multisuture craniosynostosis with turribrachycephaly. Gyral remodeling of the inner table ("copper beaten" appearance) was appreciated on both modalities and reflective of increased intracranial pressure. Intracranial malformations were more fully depicted on MR imaging (On-line Fig 3).

Patient 3 was a 2 -year-old girl with achondroplasia. CT and ZTE both demonstrated the characteristic J-shaped sella and foramen magnum stenosis. Intracranial abnormalities were more completely imaged on MR imaging (On-line Fig 4).

Patient 4 was a 6-year-old boy with Saethre-Chotzen syndrome. MR imaging was degraded by severe patient motion. Nevertheless, CT and ZTE both demonstrated craniocervical segmentation abnormality with anterior homeotic transformation and basilar impression (On-line Fig 5).

Patient 5 was a 7-month-old boy with hydrocephalus postshunting with worsening posterior plagiocephaly. MR imaging was performed at $1.5 \mathrm{~T}$ for the patient's MR imaging-compatible Strata shunt (Medtronic, Minneapolis, Minnesota). CT and ZTE both demonstrated the course of the shunt catheter. There was secondary flattening of the right parietal calvaria subjacent to the shunt valve, without synostosis. No appreciable difference in diagnostic image quality was observed on the $1.5 \mathrm{~T}$ scan. Brain abnormalities were more completely characterized on MR imaging than on CT (On-line Fig 6).

Patient 6 was a 15-month-old girl with Shprintzen-Goldberg syndrome postshunting. MR imaging was performed at $1.5 \mathrm{~T}$ for the patient's MR imaging-compatible Strata shunt. CT and ZTE both imaged the entirety of the shunt catheter. Multiple craniofacial anomalies were imaged, including pancraniosynostosis, severe turribrachycephaly, copper beaten skull, midface hypoplasia, exorbitism, and basilar impression. No appreciable difference in diagnostic image quality was observed on the $1.5 \mathrm{~T}$ scan. Intracranial malformations were better evaluated on MR imaging than on CT (On-line Fig 7).

Patient 7 was a 7 -month-old boy with atraumatic head deformity. CT and ZTE revealed a Galassi type III arachnoid cyst with associated calvarial remodeling and intracranial midline shift (On-line Fig 8).

Patient 8 was a 1 -year-old boy with a remote history of a fall and worsening head deformity. CT and ZTE equivalently demonstrated a leptomeningeal cyst or growing fracture of the right parietal calvaria with underlying parenchymal encephalomalacia. This presumably represented expansion of a remote skull fracture with dural injury and chronic CSF pulsations (On-line Fig 9).

Patient 9 was an 8-year-old boy with chronic epilepsy on anticonvulsants. CT and ZTE equivalently demonstrated diffuse hyperostosis with normal sutural, sinonasal, and temporal bone anatomy for his age. Brain abnormalities were better depicted on MR imaging (On-line Fig 10).

Patient 10 was a 2-month-old girl with breathing difficulties. CT and ZTE both showed bilateral choanal stenosis with inward bowing of the posterior nasal walls. Brain abnormalities were more readily evaluated on MR imaging (On-line Fig 11).

Patient 11 was a 17 -year-old girl with neurofibromatosis type 1. MR imaging demonstrated orbitotemporal neurofibromatosis with left proptosis and transspatial plexiform neurofibroma. ZTE confirmed characteristic bone remodeling with expansion of the left bony orbit and sphenoid wing dysplasia. Soft-tissue findings were better depicted on MR imaging (Online Fig 12).

Patient 12 was an 11-year-old girl with right facial pain and swelling. CT and ZTE both depicted erosion of the right pterygoid plate secondary to a right masticator space mass, biopsied as alveolar rhabdomyosarcoma. Additional soft-tissue detail was obtained using MR imaging (On-line Fig 13).

Patient 13 was a 6-year-old boy post-calvarial reconstruction with a polyether etherketone implant. CT and ZTE comparably showed the morphology of the cranioplasty and fixating hardware, with minimal image artifacts (On-line Fig 14).

Patient 14 was a 9-year-old girl with facial trauma due to a motor vehicle collision. Preoperative CT demonstrated a mildly displaced left zygomaticomaxillary fracture. Following surgical fixation, ZTE was performed and confirmed anatomic realignment of fracture fragments by fixating hardware, with minimal image artifacts (On-line Fig 15). 


\section{DISCUSSION}

Zero TE is the newest in a series of short-TE approaches, including gradient-echo and ultrashort-echo techniques, that can successfully image short-T2 structures including cortical bone, meninges, cartilage, tendons, ligaments, calcium, airway, and lungs. ${ }^{3-6}$ Additional intrinsic sequence properties include rapid imaging times, silent scanning, high-resolution isotropic datasets, and resistance to artifacts. ${ }^{7-9}$ The applicability of ZTE for bone imaging has been successfully demonstrated in small clinical case series and ex vivo studies of the cranial vault, face, jaw, and spine. ${ }^{12-}$

${ }^{19}$ Because MR imaging already provides superior soft-tissue detail, the addition of a ZTE bone sequence would enable highly efficient and radiation-free evaluation in several pediatric neuroimaging, head/neck imaging, and musculoskeletal imaging scenarios. Inversion recovery techniques can also yield T1-weighted ZTE images useful for assessing brain and spinal cord myelination, tumor and pituitary imaging, vessels, and airway. ${ }^{22-24}$ Additional potential applications include 3D modeling and printing, metal artifact reduction, fetal imaging, PET/MRI attenuation correction, radiation therapy planning, and interventional guidance. ${ }^{25-29}$

The limitations to wider implementation of ZTE are multifactorial and include physician awareness, scanner and hardware compatibility, vendor sequence availability, clinical workflow, and image postprocessing and analysis. Currently, there is a major knowledge gap regarding the diagnostic parallels between ZTE and CT. Our preliminary data illustrate that the 2 modalities provide qualitatively similar bone detail across a wide variety of neuroimaging pathologies, without any cases of diagnostic discordance. To definitively evaluate the clinical utility of ZTE, we are currently conducting a well-powered and controlled clinical trial to quantitatively evaluate the comparative effectiveness of these 2 modalities. Our hypothesis is that ZTE is equivalent to CT for neuroanatomic landmark visualization across the life span, enables imaging diagnosis across a variety of pathologies, and permits effective anatomic modeling and interventional planning. In addition, we are investigating improved techniques for pseudoCT and $3 \mathrm{D}$ reconstructions to facilitate diagnosis. Current vendor-available automated algorithms do not consistently correct for the variable intensities of developing bone, cartilage, soft tissues, and airway secretions, creating diagnostic challenges in the pediatric population. At present, we are working on a semiautomated postprocessing technique $^{20}$ and investigating machine learning approaches to generate improved pseudoCT images. ${ }^{29,30}$

\section{CONCLUSIONS}

ZTE is a promising technique for radiation-free bone imaging with rapid, high-resolution, silent, and artifact-resistant properties. Although larger studies will be needed, our preliminary experience demonstrates the diagnostic utility and clinical applications of this technique for radiation-free bone imaging.

\section{ACKNOWLEDGMENTS}

The authors wish to thank Uldis Bite, MD, and Samir Mardini, $\mathrm{MD}$, for their clinical support of this work; and Norbert G. Campeau, MD, for assistance with On-Line Figure 2E.
Disclosures: Mai-Lan Ho-RELATED: Grant: Radiological Society of North America, Society for Pediatric Radiology, American Society of Head and Neck Radiology, Comments: Principal Investigator on research grants*; UNRELATED: Payment for Lectures Including Service on Speakers Bureaus: American Roentgen Ray Society, Comments: honoraria for lectures; Royalties: McGraw-Hill, Comments: book royalties. *Money paid to the institution.

\section{REFERENCES}

1. Risk of ionizing radiation exposure to children: a subject review. American Academy of Pediatrics-Committee on Environmental Health. Pediatrics 1998;101(4 Pt 1):717-19 Medline

2. Frush DP, Goske MJ. Image gently: toward optimizing the practice of pediatric CT through resources and dialogue. Pediatr Radiology 2015;45:471-75 CrossRef Medline

3. Weiger M, Pruessmann KP, Hennel F. MRI with zero echo time: hard versus sweep pulse excitation. Magn Reson Med 2011;66:37989 CrossRef Medline

4. Schieban $\mathrm{K}$, Weiger M, Hennel F, et al. ZTE imaging with enhanced flip angle using modulated excitation. Magn Reson Med 2015; 74:684-93 CrossRef Medline

5. Weiger M, Wu M, Wurnig MC, et al. ZTE imaging with long-T2 suppression. NMR Biomed 2015;28:247-54 CrossRef Medline

6. Froidevaux R, Weiger M, Brunner DO, et al. Filling the dead-time gap in zero echo time MRI: principles compared. Magn Reson Med 2018;79:2036-45 CrossRef Medline

7. Alibek S, Vogel M, Sun W, et al. Acoustic noise reduction in MRI using Silent Scan: an initial experience. Diagn Interv Radiology 2014;20:360-63 CrossRef Medline

8. Ognard J, Burdin V, Ragoubi Hor R, et al. "Bones in Silenz": a new T1-weighted SILENZ sequence evaluating the bone in MRI. Diagn Interv Imaging 2015;96:973-75 CrossRef Medline

9. Tiberi G, Costagli M, Biagi L, et al. SAR prediction in adults and children by combining measured B1+ maps and simulations at 7.0 Tesla. J Magn Reson Imaging 2016;44:1048-55 CrossRef Medline

10. Zheng W, Kim JP, Kadbi M, et al. Magnetic resonance-based automatic air segmentation for generation of synthetic computed tomography scans in the head region. Int J Radiat Oncol Biol Phys 2015;93:497-506 CrossRef

11. Delso G, Wiesinger F, Sacolick LI, et al. Clinical evaluation of zeroecho-time MR imaging for the segmentation of the skull. $\mathrm{J} \mathrm{Nucl}$ Med 2015;56:417-22 CrossRef Medline

12. Eley KA, Watt-Smith SR, Sheerin F, et al. "Black Bone" MRI: a potential alternative to $\mathrm{CT}$ with three-dimensional reconstruction of the craniofacial skeleton in the diagnosis of craniosynostosis. Eur Radiol 2014;24:2417-26 CrossRef

13. Eley KA, Watt-Smith SR, Golding SJ. "Black bone" MRI: a potential alternative to CT when imaging the head and neck: report of eight clinical cases and review of the Oxford experience. BJR 2012; 85:1457-64 CrossRef

14. Naganawa S, Nakane T, Kawai $H$, et al. Visualization of middle ear ossicles in elder subjects with ultra-short echo time MR imaging. Magn Reson Med Sci 2017;16:93-97 CrossRef Medline

15. Kobayashi N, Goerke U, Wang L, et al. Gradient-modulated PETRA MRI. Tomography 2015;1:85-90 CrossRef Medline

16. Hövener JB, Zwick S, Leupold J, et al. Dental MRI: imaging of soft and solid components without ionizing radiation. J Magn Reson Imaging 2012;36:841-46 CrossRef Medline

17. Koo TK, Kwok WE. A non-ionizing technique for three-dimensional measurement of the lumbar spine. J Biomech 2016;49:407379 CrossRef

18. Dremmen MHG, Wagner MW, Bosemani T, et al. Does the addition of a "black bone" sequence to a fast multisequence trauma MR protocol allow MRI to replace CT after traumatic brain injury in children? AJNR Am J Neuroradiol 2017;38:2187-92 CrossRef Medline 
19. Weiger M, Stampanoni M, Pruessmann KP. Direct depiction of bone microstructure using MRI with zero echo time. Bone 2013;54:44-47 CrossRef Medline

20. Lu A, Gorny KR, Ho ML, et al. Improved delineation of air-bone interface in in-vivo high-resolution bright bone ZTE MRI at 3T. In: Proceedings of the Annual Meeting of the International Society for Magnetic Resonance in Medicine. Honolulu, Hawaii. April 22-27, 2017 P5109

21. Wehrli FW. Magnetic resonance of calcified tissues. J Magn Reson 2013;229:35-48 CrossRef Medline

22. Weiger M, Brunner DO, Dietrich BE, et al. ZTE imaging in humans. Magn Reson Med 2013;70:328-32 CrossRef Medline

23. Holdsworth SJ, Macpherson SJ, Yeom KW, et al. Clinical evaluation of silent T1-weighted MRI and silent MR angiography of the brain. AJR Am J Roentgenol 2018;210:404-11 CrossRef Medline.

24. Niwa T, Nozawa K, Aida N. Visualization of the airway in infants with MRI using pointwise encoding time reduction with radial acquisition (PETRA). J Magn Reson Imaging 2017;45:839-44 CrossRef Medline
25. Eley KA, Watt-Smith SR, Golding SJ. "Black Bone" MRI: a novel imaging technique for 3D printing. Dentomaxillofac Radiol 2017; 46:20160407 CrossRef

26. Hilgenfeld T, Prager M, Heil A, et al. PETRA, MSVAT-SPACE and SEMAC sequences for metal artefact reduction in dental MR imaging. Eur Radiol 2017;27:5104-12 CrossRef

27. Robinson AJ, Blaser S, Vladimirov A, et al. Foetal "black bone" MRI: utility in assessment of the foetal spine. Br J Radiology 2015;88:20140496 CrossRef Medline

28. Himes NC, Chansakul T, Lee TC. Magnetic resonance imaging-guided spine interventions. Magn Reson Imaging Clin $\mathrm{N}$ Am 2015;23:523-32 CrossRef

29. Wiesinger F, Bylund M, Yang J, et al. Zero TE-based pseudo-CT image conversion in the head and its application in PET/MR attenuation correction and MR-guided radiation therapy planning. Magn Reson Med 2018;80:1440-51 CrossRef

30. Dinkla AM, Wolterink JM, Maspero M, et al. MR-only brain radiation therapy: dosimetric evaluation of synthetic CTs generated by a dilated convolutional neural network. Int J Radiat Oncol Biol Phys 2018;102:801-12 CrossRef 